\title{
Implementation of an Oral Care Protocol for Primary Diabetes Care: A Pilot Cluster-Randomized Controlled Trial
}

\author{
Martijn J. L. Verbulst, $P b D, M S c^{1}$ \\ Wijnand J. Teeuw, PbD, MSc, DDS ${ }^{1}$ \\ Victor E. A. Gerdes, PbD, MD ${ }^{2,3}$ \\ Bruno G. Loos, PbD, MSc, DDS ${ }^{1}$ \\ 'Department of Periodontology, Academic \\ Centre for Dentistry Amsterdam, Univer- \\ sity of Amsterdam and Vrije Universiteit, \\ Amsterdam, The Netherlands \\ ${ }^{2}$ Department of Vascular Medicine, \\ Amsterdam University Medical Centers, \\ Amsterdam, The Netherlands \\ ${ }^{3}$ Department of Internal Medicine, Spaarne \\ Gasthuis, Hoofddorp, The Netherlands
}

Conflicts of interest: authors report none.

\section{CORRESPONDING AUTHOR}

Martijn J. L. Verhulst

Department of Periodontology, Academic Centre for Dentistry Amsterdam, University of Amsterdam and Vrije Universiteit Gustav Mahlerlaan 3004 1081 LA Amsterdam, The Netherlands paro@acta.nl

\begin{abstract}
PURPOSE Although diabetes care guidelines recommend paying attention to oral health, the effect on daily practice has been limited, and patients with diabetes have yet to benefit. We investigated whether implementation of an oral care protocol for general practitioners (GPs [family physicians]) can improve patientcentered outcomes for patients with type 2 diabetes.
\end{abstract}

METHODS Twenty-four GP offices were randomly assigned to the experimental or control group (12 offices each). In the experimental group, GPs and nurse practitioners implemented an oral care protocol. No extra attention was given to oral health in the control group. The primary outcome parameter was oral health-related quality of life (QoL) assessed with the 14-item Oral Health Impact Profile at baseline and 1 year later. Other outcomes were self-reported oral health complaints and general health-related QoL (36-item Short Form Health Survey).

RESULTS Of 764 patients with type 2 diabetes, 543 (71.1\%) completed the 1-year follow-up. More patients reported improved oral health-related QoL in the experimental group (35.2\%) compared to the control group (25.9\%) $\left(P=.046 ; P_{\text {adj }}=.049\right)$. In a secondary post hoc analysis including GP offices with $\geq 60 \%$ patient follow-up $(n=18)$, improvement was $38.3 \%$ and $24.9 \%$, respectively ( $P$ and $\left.P_{\text {adj }}=.011\right)$. Improvement of self-reported oral health complaints did not differ between groups. The intervention had no effect on general healthrelated QoL, with the exception of the concept scale score for changes in health over time $\left(P_{\text {adj }}=.033\right)$.

CONCLUSIONS Implementation of an oral care protocol in primary diabetes care improved oral health-related QoL in patients with type 2 diabetes.

Ann Fam Med 2021;19:197-206. https://doi.org/10.1370/afm.2645.

\section{INTRODUCTION}

$\mathrm{D}$ iabetes mellitus is a disease of epidemic proportions, currently affecting 451 million individuals worldwide. ${ }^{1}$ The most devastating consequence of diabetes mellitus is the development of chronic complications, such as retinopathy, nephropathy, neuropathy, and cardiovascular disease, ${ }^{2}$ and their detrimental effects on quality of life (QoL). ${ }^{3,4}$ Since the recognition that periodontitis, which involves inflammation of the supporting structures of the teeth (root cementum, periodontal ligament, alveolar bone), is another complication of diabetes mellitus, ${ }^{5}$ awareness of the need for integrated dental-medical health care for patients with diabetes mellitus has started to increase ${ }^{6}$ Periodontitis can also negatively influence metabolic control, ${ }^{7}$ and research has shown that intensive treatment of periodontitis improves metabolic control in patients with diabetes mellitus. ${ }^{8,9}$ Other oral diseases and complaints, such as dry mouth (xerostomia), Candida infections, taste disorders, and oral (pre)malignancies, also appear to be more prevalent in patients with diabetes mellitus. ${ }^{10}$

The accumulation of evidence on the bidirectional association between diabetes mellitus and oral health has resulted in several recommendations 
and guidelines for health care professionals. ${ }^{11-14}$ One of the key messages is to ask patients with diabetes mellitus about dentist visits and to motivate those individuals who do not visit a dentist regularly to do so. Patients should also be made aware that attention to oral hygiene is a basic aspect of diabetes mellitus selfmanagement. ${ }^{12}$ Although implementation of oral health in diabetes care is advised, the effectiveness of such implementation has not been studied. The abundance of information-increasing awareness and knowledge about oral health among diabetes care professionalshas had limited effect on diabetes care thus far. ${ }^{15-18}$ The medical and dental professions generally act as segregated disciplines, and as a result, it is likely that many patients with diabetes mellitus do not receive the optimal oral care that they might need.

In the Netherlands, primary diabetes care is organized within a structured, interdisciplinary program, with central roles for general practitioners (GPs [family physicians]) and specialized nurse practitioners. This program is covered by basic health care insurance with a deductible, which is mandatory for the entire population. However, oral care is not included and is covered only on the basis of voluntary dental insurance packages. Modern primary diabetes care programs, such as that in the Netherlands, aim to prevent diabetes complications and improve QoL. ${ }^{19}$ The primary aim of the present study was to assess whether an oral health protocol implemented at the level of the GP office can improve oral health-related QoL. We also assessed improvements in specific self-reported oral health complaints and changes in general health-related QoL.

\section{METHODS}

\section{Study Design}

The study design of this cluster-randomized controlled trial has been described elsewhere. ${ }^{20}$ In brief, GP offices were recruited by the principal investigator (M.J.L.V.). There were no eligibility criteria for the GP offices, other than being situated in the city or area of Amsterdam. Interviews with GP offices were planned at their convenience. After accepting to participate, GP offices were assigned to the experimental group or the control group with the use of a sequentially numbered, opaque, sealed envelope allocation protocol (ie, cluster level). At the participating offices, patients with type 2 diabetes mellitus were enrolled by GPs and/or nurse practitioners, depending on who was responsible for the regular diabetes consultation hours. Patient recruitment at the participating offices was not random. General practitioner office recruitment, patient eligibility criteria, and participant selection and inclusion are presented in Figure 1 and described in more detail elsewhere. ${ }^{20}$
Considering the fact that this was the first study of its kind, optimal sample size could not be determined beforehand. Therefore, this study was considered a pilot study. Study participants were followed up for 1 year. The study was approved by the Medical Ethical Committee of the Vrije Universiteit Medical Centre (2014.585) and registered at the International Standard Randomised Controlled Trial Number registry (number: ISRCTN10145611).

\section{Intervention}

The intervention was targeted to GP offices (ie, cluster level). As intervention, GPs and nurse practitioners in the experimental group were instructed to implement the following oral care protocol into their daily routine:

(1) Educate the patient about the importance of oral health and motivate them to pay attention to their oral hygiene. This was repeated at each subsequent visit.

(2) Encourage the patient to visit the dentist at regular intervals and repeat this advice at each subsequent visit. To support this, the patient received a standardized referral letter for a dentist at enrollment, which was developed for this occasion (Supplemental Appendix 1, https://www.AnnFamMed.org/content/19/3/197/ suppl/DC1/).

(3) At enrollment, provide an introduction kit containing oral hygiene products (provided by Sunstar GUM [Sunstar Suisse SA]) and an information brochure elaborating on the association between diabetes mellitus and oral health.

The GP offices in the control group were instructed to follow their standard diabetes care program, without any extra attention to oral health.

\section{Outcomes}

We evaluated the effect of the intervention (targeted to GP offices, ie, clusters) on patient-centered outcomes of oral health. Participating GPs and nurse practitioners were responsible for coordinating and performing the measurements of all outcome parameters, both at baseline and follow-up (after 1 year).

\section{Oral Health-Related QoL}

Oral health-related QoL was assessed using the Dutch version of the short-form Oral Health Impact Profile (OHIP-NL14), ${ }^{21-23}$ which consists of 14 items that inquire how often the respondent has experienced certain problems or complaints regarding oral health in the past 12 months. Answering these 14 items results in 7 conceptual subdomain scores including functional limitation, physical pain, psychologic discomfort, physical disability, psychologic disability, social disability, and handicap. The 14 items are coded on a 5-point Likert scale of never (0), hardly ever (1), occasionally 
(2), fairly often (3), and very often (4). ${ }^{21}$ Therefore, the subdomain scores range from 0 to 8 ( 2 scores of a maximum of 4 per subdomain). The total OHIP-NL14 score, created by summing all responses, ranges from 0 to 56 . A lower score indicates greater oral healthrelated QoL. Only completed questionnaires were used to calculate subdomain and total scores; those with $\geq 1$ missing items were discarded from the final analysis.

The OHIP-NL14 questionnaire required approximately 5 minutes of the patient's time and was selfadministered in the waiting room at the GP office at the moment of inclusion (baseline) and at follow-up (after 1 year). Improvement in oral health-related QoL was assessed as recommended by the original author. ${ }^{24}$ This included subtracting the baseline scores from the follow-up scores, which resulted in a net-change score. Improvement in oral health-related QoL for both the total score and subdomains was defined as an OHIPNL14 net change score $<0$.

\section{Self-Reported Oral Health Complaints}

At baseline, study participants were asked whether they had $\geq 1$ of the following oral complaints: pain in the mouth, a dry mouth, and/or bad breath. The same questions were repeated at the 12 -month follow-up. A complaint that was reported at baseline and not mentioned at follow-up was considered an improvement.

\section{General Health-Related QoL}

General health-related QoL was assessed at baseline and follow-up using the validated Dutch 36 -item Short Form Health Survey. ${ }^{25}$ This questionnaire consists of 36 items covering 8 health concept scales of physical functioning (10 items), social functioning ( 2 items), role limitations due to physical health problems (4 items), role limitations due to emotional problems (3 items), general mental health (5 items), vitality (4 items), bodily pain ( 2 items), and general health perceptions (5 items). ${ }^{26}$ In addition to these 8 concept scales, 1 item represents perceived change in health over time. ${ }^{27}$ The resulting concept scores are presented on a linear scale (0-100), with a higher score indicating greater general health-related QoL. Only completed questionnaires were used to calculate concept scale scores ; those with $\geq 1$ missing item were discarded. The questionnaire was self-administered in the waiting room at the GP office and required approximately 10 minutes of the patient's time.

\section{Statistical Analysis}

We used descriptive statistics to present the baseline patient characteristics for both groups. Independent $t$ tests and $\chi^{2}$ test were used to analyze whether patients lost to follow-up were any different from patients who completed the study. We used $\chi^{2}$ statistics to assess differences in improvement of oral health-related QoL between the experimental and control GP offices. This analysis was also performed for improvement in self-reported oral health complaints. Intracluster correlation coefficients (ICCs) were computed to adjust the $\chi^{2}$ statistics for the effect of clustering within GP offices, ${ }^{28}$ as suggested by the Consolidated Standards of Reporting Trials statement for cluster-randomized controlled trials. ${ }^{29}$ The group-specific adjustment approach proposed by Donner and Klar was used to estimate the ICCs. ${ }^{28}$ Negative ICCs were truncated to 0 because these are generally considered to be implausible in cluster-randomized trials. ${ }^{30,31}$ Differences in general health-related QoL between the experimental and control groups were analyzed using analysis of covariance adjusted for baseline general health-related QoL values. To further adjust for the possible effect of clustering within GP offices, linear mixed-model analyses were applied, with general health-related QoL as the dependent variable, group allocation (experimental/control) and baseline general health-related QoL as fixed factors, and clustering (GP office number) as a random factor. Analyses were performed using IBM SPSS Statistics version 25 (IBM Corp), and $P$ values $<.05$ were considered statistically significant.

General practitioner offices with low followup could mask the true effect of the intervention because it might indicate that GPs, nurse practitioners, and/or patients were struggling to adhere to the protocol. Therefore, we calculated the median follow-up and its interquartile range and performed secondary analyses for all outcome parameters including only patients from GP offices with a followup greater than the first quartile.

\section{RESULTS \\ Participants}

Figure 1 presents an overview of the entire study flow. Of the 49 GP offices that were invited to an admission interview during the period March 2015 to November 2015, 24 agreed to participate. At these participating offices, 764 patients with type 2 diabetes mellitus were included by GPs and nurse practitioners: 352 in the experimental group and 412 in the control group. Patient characteristics at baseline, including general demographic and health characteristics, self-reported oral health characteristics, oral health-related QoL, and general health-related $\mathrm{QoL}$, are summarized in Table 1.

Of the 764 patients, 221 (29\%) were lost to followup. A loss-to-follow-up analysis for these patients is presented in Supplemental Appendix 2 (https://www. 


\section{Figure 1. Study flowchart.}

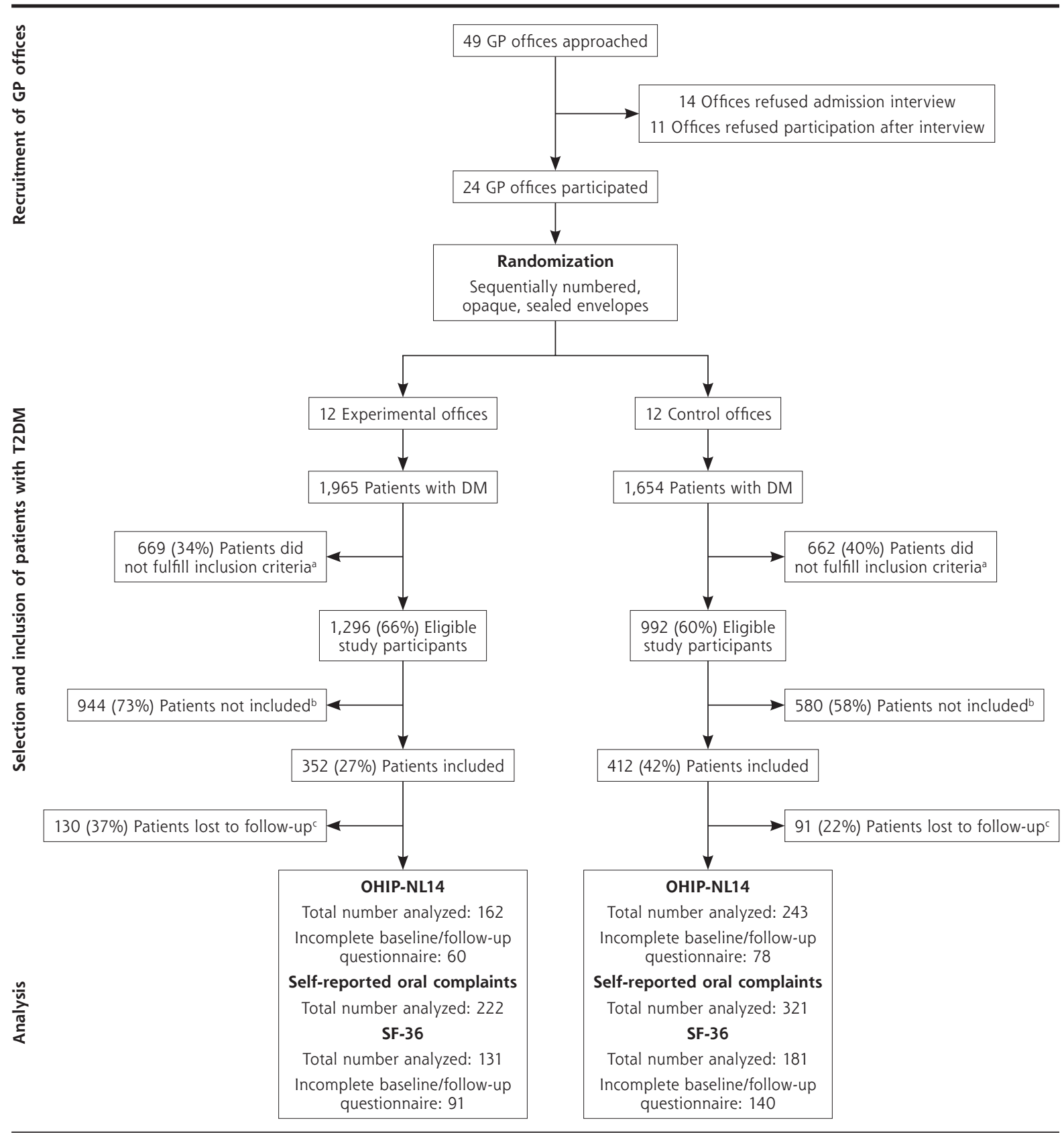

DM = diabetes mellitus; GP = general practitioner; OHIP-NL14 = 14-item Oral Health Impact Profile; SF-36 = 36-item Short Form Health Survey; T2DM = type 2 diabetes mellitus.

a Inclusion criteria: (1) aged $\geq 18$ years; (2) diagnosed with T2DM; (3) follows the standardized primary care protocol, including an annual examination; (4) understands spoken and written Dutch.

${ }^{b}$ Main reasons for not participating in the study: lack of time and routine of the nurse practitioners and/or general practitioner; financial issues for certain patients; disinterest and lack of motivation of certain patients; dental anxiety.

c Reasons for loss to follow-up: transfer of GP office $(n=27)$; renounciation from participation $(n=25)$; illness and/or weakness, eg, due to dementia or severe diabetic complications $(n=11)$; death $(n=10)$; coding mistake, ie, data assigned to follow-up rather than baseline $(n=5)$; incomplete follow-up measurements without a reason $(n=143)$.

AnnFamMed.org/content/19/3/197/suppl/DC1/). Supplemental Table 1 (https://www.AnnFamMed.org/ content/19/3/197/suppl/DC1/) summarizes patient flow for each participating GP office, listing total number of patients with diabetes mellitus, those eligible for inclusion in study, those included, and those followed up. Median follow-up was $81 \%$, with an interquartile range of $60 \%$ to $92 \%$. General practitioner offices with 
Table 1. Patient Characteristics at Baseline, by Study Group

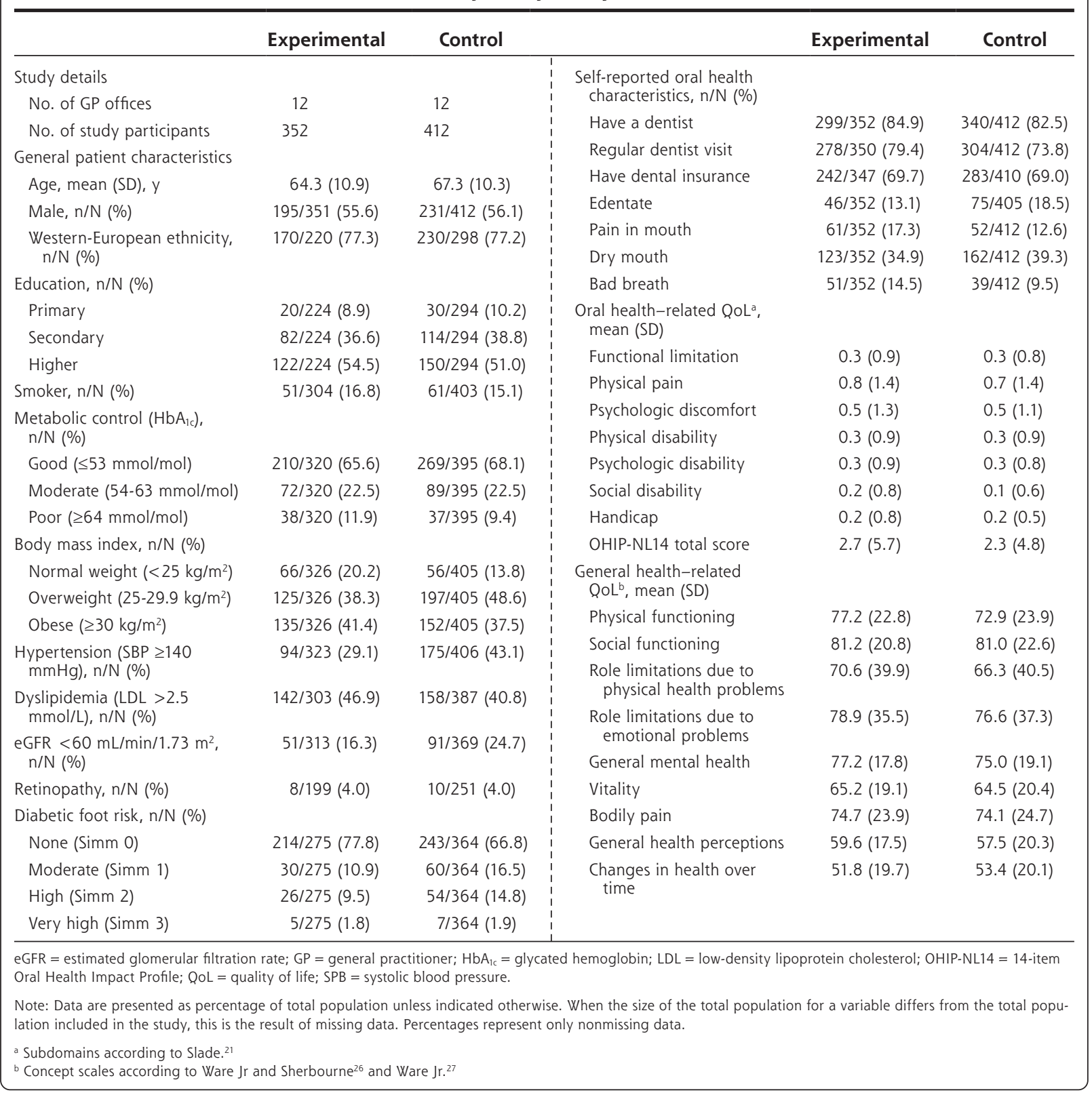

a follow-up $<60 \%$ were identified (Supplemental Table 1 ), and the patients belonging to those offices were excluded from the secondary analyses.

\section{Outcomes}

\section{Oral Health-Related QoL}

The primary analysis, which considered all participating GP offices, included 405 patients with a baseline and a follow-up measurement for oral health-related QoL (Figure 1). Improvement in total OHIP-NL14 score was greater in the experimental group (35.2\%) compared to the control group (25.9\%) (Table 2). This difference was significant $\left(\chi^{2}: 3.997 ; P=.046\right)$ and remained significant in the analysis adjusted for clustering (ICC: $0.002 ; \chi_{\text {adj: }}^{2} 3.857_{i} P_{\text {adj }}=.049$ ). The proportions of patients with an improvement in the OHIP-NL14 subscale scores psychologic disability $\left(\chi^{2}\right.$ : 4.451; $P=.035)$ and handicap $\left(\chi^{2}: 4.513 ; P=.034\right)$ were also increased in the experimental group compared to the control group. After adjusting for clustering effect, however, the difference became nonsignificant (Table 2). The secondary analysis, including only patients from GP offices with follow-up $\geq 60 \%$, included 325 patients (Table 3). In this analysis, again, substantially greater improvement in the total OHIP-NL14 score was observed in the experimental group $(38.3 \%)$ 
compared to the control group (24.9\%) (ICC: -0.005 ; $\chi^{2}$ and $\chi_{\text {adj: }}^{2}$ 6.545; $P$ and $\left.P_{\text {adj }}=.011\right)$. In this analysis, significantly more patients from experimental GP offices showed improvements in the OHIP-NL14 subscale scores of physical pain, psychologic discomfort, psychologic disability, and handicap compared to patients from GP offices in the control group. These differences were observed in the unadjusted analysis as well as in the analysis adjusted for clustering (Table $3)$. Baseline, follow-up, and change scores in oral health-related QoL for the analytic sample $(n=405)$ expressed as continuous variables are summarized in Supplemental Table 2 (https://www.AnnFamMed.org/ content/19/3/197/suppl/DC1/). Data for GP offices with follow-up $<60 \%$ are summarized in Supplemental Table 3 (https://www.AnnFamMed.org/content/19/3/197/ suppl/DC1/).

\section{Self-Reported Oral Health Complaints}

For the primary analysis, 543 patients with a baseline and a follow-up measurement for self-reported oral health complaints were analyzed (Table 2). In the unadjusted analysis, improvement in the complaint of pain in the mouth was significantly greater in the experimental group compared to the control group $\left(\chi^{2}\right.$ : 4.965; $P=.026)$. This difference became nonsignificant after adjusting for clustering $\left(\chi_{\text {adj: }}^{2}: 3.451_{i} P_{\text {adj }}=.063\right)_{\text {; }}$ improvement did not differ for any of the oral health complaints between patients from the experimental and control GP offices (Table 2). This also applied for the secondary analysis, with only patients from GP offices with follow-up $\geq 60 \%$ included in the analysis (441 patients analyzed) (Table 3 ).

\section{General Health-Related QoL}

General health-related QoL scores at baseline and follow-up are summarized in Table 4 . In the primary analysis, the intervention had no effect on any of the general health-related QoL concept scale scores, with the exception of the item changes in health over time $(P=.033)$ (Table 4$)$. This item did not change in the experimental group (mean difference: $1.0_{i} P=.659$ ) but decreased significantly in the control group (mean difference: $-5.9 ; P=.001$ ). When patients from GP offices

Table 2. Loss to Follow-Up, Improvement in Oral Health-Related QoL, and Self-Reported Oral Health Complaints by Intervention, Including All GP Offices in the Analysis

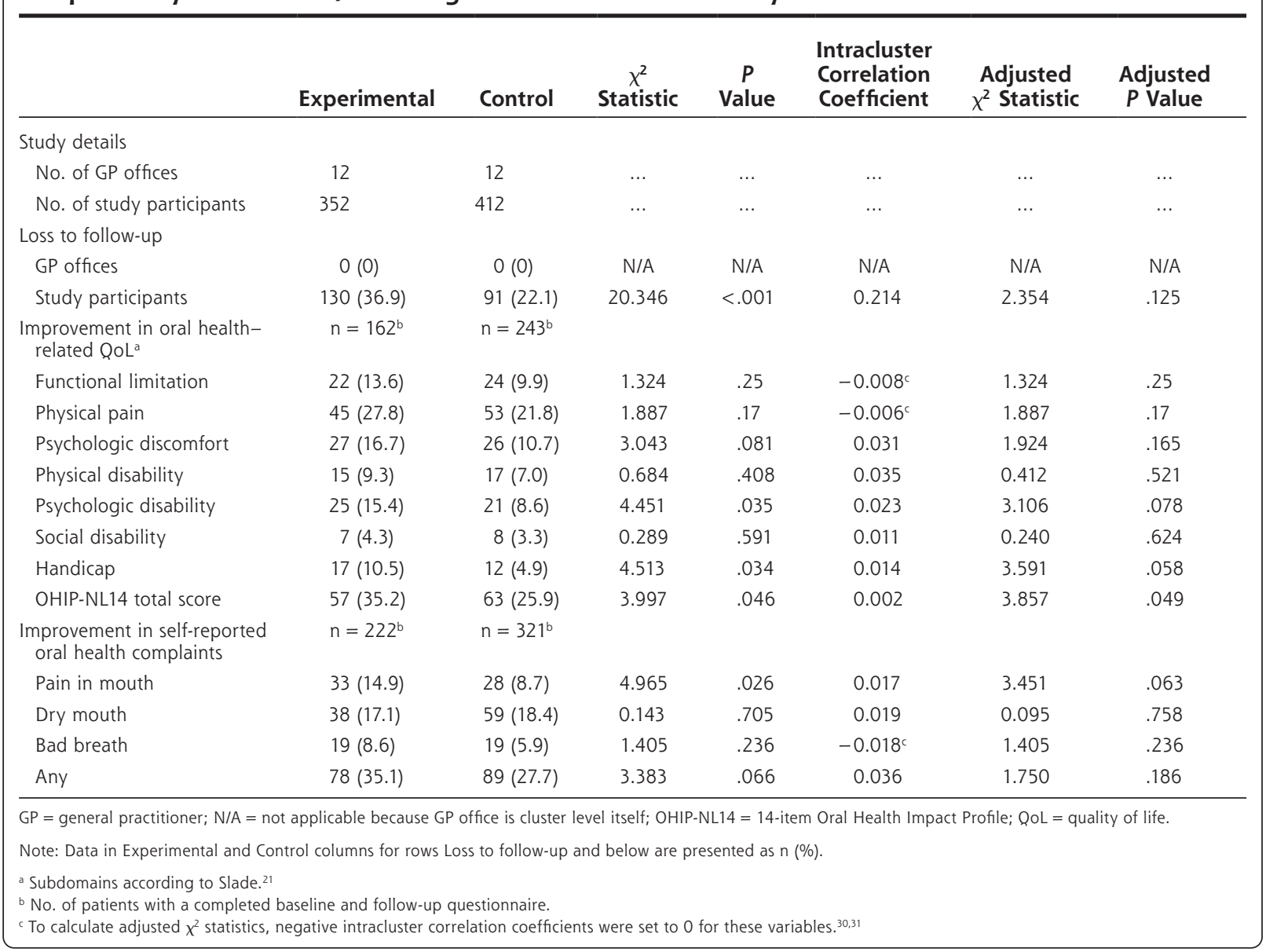


with follow-up $<60 \%$ were excluded from the analysis, none of the concept scale scores showed differences between the experimental and control groups (Table 4).

\section{DISCUSSION}

Results of the present study showed that implementation of an oral care protocol in primary diabetes care increased improvement in oral health-related QoL in patients with type 2 diabetes mellitus. This effect became stronger when the analysis focused on GP offices with high follow-up. These findings suggest that patients with type 2 diabetes mellitus who receive primary diabetes care can benefit from extra attention to oral health and further reflect the concept that oral health and general health are connected. As mentioned, patients with diabetes generally have an increased prevalence of several oral conditions. ${ }^{10}$ These will likely affect oral health-related QoL, as has been shown for periodontal disease ${ }^{32}$ and dry mouth conditions. ${ }^{33}$ Although existing guidelines advocate for the inclusion of oral health in the interdisciplinary treatment of patients with type 2 diabetes mellitus, the present study is the first to provide evidence for such an approach from a patient-centered point of view. This is particularly important because in modern diabetes care, patient-centered outcomes such as QoL are among the key treatment outcomes. ${ }^{19,34}$

In contrast to QoL, we did not observe increased improvement in specific oral health complaints, based on the 3 nonvalidated items of pain in the mouth, dry mouth, and bad breath. In hindsight, an additional question on bleeding gums would have been useful, although it is not a specific diabetes-related oral complaint.

The cross-sectional analysis of the our study population demonstrated that impaired oral health-related QoL coincided with impaired general health-related QoL. ${ }^{20}$ However, the intervention had no effect on most general health-related QoL concept scales, with the exception of changes in health over time. For this item, the patient was asked to rate their health compared to 1 year before. ${ }^{27}$ In the control group, this self-perceived change in health was less after 1 year, whereas it remained stable in the experimental group. Overall, the

Table 3. Loss to Follow-Up, Improvement in Oral Health-Related QoL, and Self-Reported Oral Health Complaints by Intervention, Excluding GP Offices With Follow-Up $<60 \%$

\begin{tabular}{|c|c|c|c|c|c|c|c|}
\hline & Experimental & Control & $\underset{\text { Statistic }}{\chi^{2}}$ & $\begin{array}{c}P \\
\text { Value }\end{array}$ & $\begin{array}{l}\text { Intracluster } \\
\text { Correlation } \\
\text { Coefficient }\end{array}$ & $\begin{array}{l}\text { Adjusted } \\
\chi^{2} \text { Statistic }\end{array}$ & $\begin{array}{l}\text { Adjusted } \\
P \text { Value }\end{array}$ \\
\hline \multicolumn{8}{|l|}{ Study details } \\
\hline No. of GP offices & 8 & 10 & $\ldots$ & $\ldots$ & $\ldots$ & $\ldots$ & $\ldots$ \\
\hline No. of study participants & 210 & 318 & $\ldots$ & $\ldots$ & $\ldots$ & $\cdots$ & $\ldots$ \\
\hline \multicolumn{8}{|l|}{ Loss to follow-up } \\
\hline GP offices & $0(0)$ & $0(0)$ & N/A & N/A & N/A & N/A & N/A \\
\hline Study participants & $39(18.6)$ & $48(15.1)$ & 1.111 & .292 & 0.067 & 0.356 & .551 \\
\hline $\begin{array}{l}\text { Improvement in oral health- } \\
\text { related } \mathrm{QoL}^{\mathrm{a}}\end{array}$ & $\mathrm{n}=120^{\mathrm{b}}$ & $\mathrm{n}=205^{\mathrm{b}}$ & & & & & \\
\hline Functional limitation & $17(14.2)$ & $17(8.3)$ & 2.788 & .095 & $-0.005^{c}$ & 2.788 & .095 \\
\hline Physical pain & $37(30.8)$ & $43(21.0)$ & 3.964 & .046 & $-0.005^{c}$ & 3.964 & .046 \\
\hline Psychologic discomfort & $21(17.5)$ & $20(9.8)$ & 4.118 & .042 & $-0.008^{c}$ & 4.118 & .042 \\
\hline Physical disability & $12(10.0)$ & $12(5.9)$ & 1.903 & .168 & 0.033 & 1.152 & .283 \\
\hline Psychologic disability & $22(18.3)$ & $17(8.3)$ & 7.226 & .007 & 0.007 & 6.355 & .012 \\
\hline Social disability & $4(3.3)$ & $8(3.9)$ & 0.069 & .793 & -0.018 & 0.069 & .793 \\
\hline Handicap & $15(12.5)$ & $10(4.9)$ & 6.193 & .013 & 0.010 & 5.173 & .023 \\
\hline OHIP-NL14 total score & $46(38.3)$ & $51(24.9)$ & 6.545 & .011 & $-0.005^{c}$ & 6.545 & .011 \\
\hline $\begin{array}{l}\text { Improvement in self-reported } \\
\text { oral health complaints }\end{array}$ & $\mathrm{n}=171^{\mathrm{b}}$ & $n=270^{b}$ & & & & & \\
\hline Pain in mouth & $26(15.2)$ & $28(10.4)$ & 2.277 & .131 & 0.011 & 1.764 & .184 \\
\hline Dry mouth & $26(15.2)$ & $56(20.7)$ & 2.120 & .145 & $-0.007 c$ & 2.120 & .145 \\
\hline Bad breath & $16(9.4)$ & $16(5.9)$ & 1.831 & .176 & $-0.030^{c}$ & 1.831 & .176 \\
\hline Any & $59(34.5)$ & $85(31.5)$ & 0.436 & .509 & $-0.009 c$ & 0.436 & .509 \\
\hline $\begin{array}{l}\text { GP = general practitioner; N/A = no } \\
\text { Note: Data in Experimental and Con } \\
\text { a Subdomains according to Slade. }{ }^{21} \\
\text { b Number of patients with a comple } \\
\text { ' To calculate adjusted } \chi^{2} \text { statistics, } n\end{array}$ & $\begin{array}{l}\text { applicable because } \mathrm{G} \\
\text { rol columns from Los } \\
\text { d baseline and follon } \\
\text { gative intracluster co }\end{array}$ & $\begin{array}{l}\text { folice is cluster } \\
\text { follow-up do } \\
\text { lation questionnair } \\
\text { laefficie }\end{array}$ & $\begin{array}{l}\text { /el itself; OHIP } \\
\text { are presented }\end{array}$ & $\begin{array}{l}-14=14-i t \\
n(\%) .\end{array}$ & Oral Health Impac & Profile; QoL = qua & of life. \\
\hline
\end{tabular}


Table 4. Baseline and Follow-Up Scores for General Health-Related Quality of Life and Adjusted and Unadjusted Analysis of Differences Between Patients From Experimental and Control GP Offices

\begin{tabular}{|c|c|c|c|c|c|c|}
\hline \multirow[b]{2}{*}{ SF-36 Concept Scale ${ }^{a}$} & \multicolumn{3}{|c|}{ Experimental } & \multicolumn{3}{|c|}{ Control } \\
\hline & $\begin{array}{l}\text { Baseline } \\
(n=258)^{b}\end{array}$ & $\begin{array}{l}\text { Follow-Up } \\
(n=163)^{c}\end{array}$ & $\begin{array}{l}\text { Mean } \\
\text { Difference } \\
(n=131)^{d}\end{array}$ & $\begin{array}{l}\text { Baseline } \\
(n=286)^{b}\end{array}$ & $\begin{array}{l}\text { Follow-Up } \\
(n=231)^{c}\end{array}$ & $\begin{array}{l}\text { Mean } \\
\text { Difference } \\
(n=181)^{d}\end{array}$ \\
\hline Physical functioning & $77.2(22.8)$ & $75.1(27.3)$ & $0.1(16.6)$ & $72.9(23.9)$ & $74.5(24.4)$ & $-1.4(17.8)$ \\
\hline Social functioning & $81.2(20.8)$ & $81.3(24.4)$ & $-0.2(22.5)$ & $81.0(22.6)$ & $82.0(20.5)$ & $-2.2(21.3)$ \\
\hline Role limitations due to physical health problems & $70.6(39.9)$ & $70.1(40.2)$ & $3.4(34.5)$ & $66.3(40.5)$ & $70.8(39.4)$ & $-0.8(37.0)$ \\
\hline Role limitations due to emotional problems & $78.9(35.5)$ & $80.2(35.6)$ & $3.6(39.3)$ & $76.6(37.3)$ & $79.4(34.6)$ & $-0.2(40.4)$ \\
\hline General mental health & $77.2(17.8)$ & $79.7(17.6)$ & $1.4(12.9)$ & $75.0(19.1)$ & $77.9(17.8)$ & $1.4(14.8)$ \\
\hline Vitality & $65.2(19.1)$ & $66.2(19.1)$ & $1.3(14.6)$ & $64.5(20.4)$ & $65.3(19.7)$ & $0.03(16.1)$ \\
\hline Bodily pain & $74.7(23.9)$ & $76.4(24.3)$ & $2.1(23.3)$ & $74.1(24.7)$ & $76.2(24.7)$ & $0.02(23.1)$ \\
\hline General health perceptions & $59.6(17.5)$ & $59.9(18.5)$ & $0.8(15.9)$ & $57.5(20.3)$ & $56.9(19.1)$ & $-1.8(18.7)$ \\
\hline Changes in health over time & $51.8(19.7)$ & $51.4(19.5)$ & $1.0(24.7)$ & $53.4(20.1)$ & $49.0(19.3)$ & $-5.9(23.3)$ \\
\hline \multicolumn{7}{|c|}{ ANCOVA $=$ analysis of covariance; GP $=$ general practitioner; $\mathrm{QoL}=$ quality of life; SF-36 $=36$-item Short Form Health Survey. } \\
\hline \multicolumn{7}{|c|}{ Note: Data are presented as mean (SD) for general health-related QoL scores (range 0-100). } \\
\hline \multicolumn{7}{|c|}{ 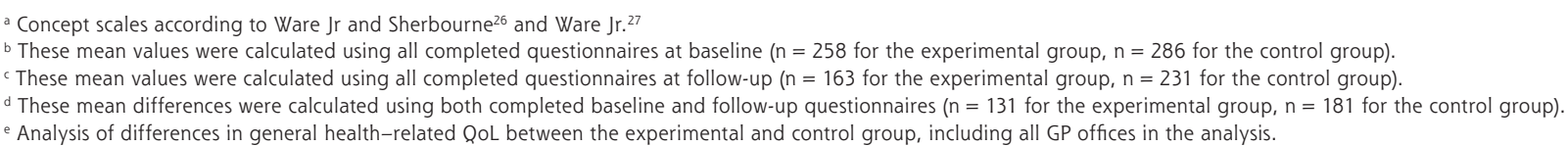 } \\
\hline
\end{tabular}

results suggest that the effects on oral health-related QoL in our study were too limited or specific to have a major effect on general health-related QoL.

The GPs and nurse practitioners in the control group were instructed not to pay more attention to oral health than usual. However, during the admission interview preceding group allocation, each GP office received the same amount of information about the background and aim of the study so that they could make a well-considered decision regarding participation. This might have introduced the Hawthorne effect to GPs and nurse practitioners allocated to the control $\operatorname{group}_{i}{ }^{35}$ they could have been motivated, be it unintentionally, to pay more attention to oral health than before the start of the study, despite being instructed not to do so. The same applies to patients in the control group, who might have been motivated by the questionnaires regarding oral health and oral health-related QoL. As a result, the effect of the intervention, compared to usual care, could have been underestimated because the control group (both at the GP office and patient levels) did not behave as they normally would have. Despite these limitations, differences in our primary outcome (oral health-related QoL) were still observed between the experimental and control group.

Because patient recruitment was performed conveniently (ie, when there was time) rather than with consecutive patients, complex cases demanding much attention might not have been included. As a result, the study population could have consisted of relatively motivated individuals. This is reflected by the relatively large proportion of patients who reported visits to the dentist at least once a year $(>75 \%)$, whereas many other studies have shown that patients with diabetes mellitus are less likely to visit a dentist regularly. ${ }^{36-39}$ This discrepancy could also be explained by the high density of dental offices in the region of Amsterdam (compared with the national average) or by the possibility that patients might have been tempted to provide socially desirable answers.

In conclusion, implementation of oral care in primary diabetes care improved oral health-related QoL in patients with type 2 diabetes mellitus. The fact that a relatively simple intervention can contribute to improvement in oral health-related QoL provides further support for the generally accepted guidelines that oral health assessment and interventions need to be included in the interdisciplinary approach for patients with type 2 diabetes mellitus in primary care.

To read or post commentaries in response to this article, go to https://www.AnnFamMed.org/content/19/3/197/tab-e-letters.

Funding support: This work was supported by Sunstar Suisse SA, Etoy, Switzerland, which had no role in the design of the study or the collection, analysis, or interpretation of the data or in writing the manuscript. 


\begin{tabular}{|c|c|c|c|}
\hline \multicolumn{2}{|c|}{ Primary Analysis ${ }^{e}$} & \multicolumn{2}{|c|}{ Secondary Analysis ${ }^{f}$} \\
\hline $\begin{array}{c}\text { Unadjusted } \\
P \text { Value }^{g}\end{array}$ & $\begin{array}{l}\text { Adjusted } \\
P \text { Value }^{\mathrm{h}}\end{array}$ & $\begin{array}{l}\text { Unadjusted } \\
P \text { Value }^{g}\end{array}$ & $\begin{array}{l}\text { Adjusted } \\
P \text { Value }\end{array}$ \\
\hline .425 & .541 & .633 & .779 \\
\hline .496 & .518 & .844 & .865 \\
\hline .371 & .465 & .458 & .566 \\
\hline .376 & .376 & .34 & .34 \\
\hline .676 & .676 & .959 & .959 \\
\hline .509 & .508 & .715 & .887 \\
\hline .513 & .513 & .669 & .669 \\
\hline .084 & .084 & .272 & .272 \\
\hline .023 & .033 & .035 & .067 \\
\hline
\end{tabular}

${ }^{f}$ Analysis of differences in general health-related QoL between the experimental and control group, excluding patients from GP offices with follow-up $<60 \%$. $g$ The unadjusted $P$ values were calculated with ANCOVA, using baseline general health-related QoL scores as covariate.

${ }^{\mathrm{h}}$ The adjusted $P$ values were calculated with linear mixed-model analysis using baseline general health-related QoL scores and group allocation (experimental/ control) as fixed factor and office number as random factor.

In addition, author Bruno G. Loos was supported in part by a grant from the University of Amsterdam for the focal point of oral infection and inflammation.

Key words: type 2 diabetes mellitus; primary care; oral care; oral health; quality of life

Submitted September 5, 2019; submitted, revised, July 19, 2020; accepted August 27, 2020.

Previous presentations: Preliminary results were presented at the CEDIADR/NOF Oral Health Research Congress, September 21, 2017, Vienna, Austria and at EuroPerio9, June 21, 2018, Amsterdam, The Netherlands.

Acknowledgments: We gratefully thank the GP offices for their participation in this study.

Supplemental materials: Available at https://www.AnnFamMed. org/content/19/3/197/suppl/DC1/.

\section{References}

1. Cho NH, Shaw JE, Karuranga S, et al. IDF Diabetes Atlas: global estimates of diabetes prevalence for 2017 and projections for 2045. Diabetes Res Clin Pract. 2018;138:271-281.

2. Fowler MJ. Microvascular and macrovascular complications of diabetes. Clin Diabetes. 2008;26(2):77-82.

3. Lloyd A, Sawyer W, Hopkinson P. Impact of long-term complications on quality of life in patients with type 2 diabetes not using insulin. Value Health. 2001;4(5):392-400.

4. U.K. Prospective Diabetes Study Group. Quality of life in type 2 diabetic patients is affected by complications but not by intensive policies to improve blood glucose or blood pressure control (UKPDS 37). Diabetes Care. 1999;22(7):1125-1136.

5. Löe H. Periodontal disease. The sixth complication of diabetes mellitus. Diabetes Care. 1993;16(1):329-334.
6. Lalla E, Papapanou PN. Diabetes mellitus and periodontitis: a tale of two common interrelated diseases. Nat Rev Endocrinol. 2011; 7(12):738-748.

7. Preshaw PM, Alba AL, Herrera D, et al. Periodontitis and diabetes: a two-way relationship. Diabetologia. 2012;55(1):21-31.

8. D'Aiuto F, Gkranias N, Bhowruth D, et al; TASTE Group. Systemic effects of periodontitis treatment in patients with type 2 diabetes: a 12 month, single-centre, investigator-masked, randomised trial. Lancet Diabetes Endocrinol. 2018;6(12):954-965.

9. Madianos PN, Koromantzos PA. An update of the evidence on the potential impact of periodontal therapy on diabetes outcomes. J Clin Periodontol. 2018;45(2):188-195.

10. Verhulst MJL, Loos BG, Gerdes VEA, Teeuw WJ. Evaluating all potential oral complications of diabetes mellitus. Front Endocrinol (Lausanne). 2019;10:56.

11. American Diabetes Association. Diabetes and oral health. Accessed Mar 26, 2021. https://professional.diabetes.org/sites/professional. diabetes.org/files/pel/source/sci-advisor_2018_diabetes_oral_ health_v4.pdf

12. International Diabetes Federation. Guideline on oral health for people with diabetes. International Diabetes Federation. 2009.

13. Sanz M, Ceriello A, Buysschaert M, et al. Scientific evidence on the links between periodontal diseases and diabetes: consensus report and guidelines of the joint workshop on periodontal diseases and diabetes by the International Diabetes Federation and the European Federation of Periodontology. Diabetes Res Clin Pract. 2018; 137:231-241.

14. National Diabetes Education Program. Pharmacy, Podiatry, Optometry, and Dental Professionals Work Group. Working Together to Manage Diabetes: A Guide for Pharmacy, Podiatry, Ooptometry, and Dental Professionals. US Centers for Disease Control and Prevention; 2012.

15. Bissett SM, Stone KM, Rapley T, Preshaw PM. An exploratory qualitative interview study about collaboration between medicine and dentistry in relation to diabetes management. BMJ Open. 2013;3(2): e002192.

16. Poudel P, Griffiths R, Wong VW, Arora A, George A. Knowledge and practices of diabetes care providers in oral health care and their potential role in oral health promotion: a scoping review. Diabetes Res Clin Pract. 2017;130:266-277.

17. Poudel P, Griffiths R, Wong VW, et al. Perceptions and practices of general practitioners on providing oral health care to people with diabetes - a qualitative study. BMC Fam Pract. 2020;21(1):34.

18. Tse SY. Diabetes mellitus and periodontal disease: awareness and practice among doctors working in public general out-patient clinics in Kowloon West Cluster of Hong Kong. BMC Fam Pract. 2018; 19(1):199.

19. Rutten G, de Grauw W, Nijpels G, et al. NHG-Standaard Diabetes mellitus type 2 (derde herziening). Huisarts Wet. 2013;56(10):512525. [Article in Dutch]

20. Verhulst MJ, Teeuw WJ, Gerdes VE, Loos BG. Self-reported oral health and quality of life in patients with type 2 diabetes mellitus in primary care: a multi-center cross-sectional study. Diabetes Metab Syndr Obes. 2019;12:883-899.

21. Slade GD. Derivation and validation of a short-form oral health impact profile. Community Dent Oral Epidemiol. 1997;25(4):284-290.

22. van der Meulen MJ, John MT, Naeije M, Lobbezoo F. The Dutch version of the Oral Health Impact Profile (OHIP-NL): translation, reliability and construct validity. BMC Oral Health. 2008;8:11.

23. van der Meulen MJ, John MT, Naeije M, Lobbezoo F. Developing abbreviated OHIP versions for use with TMD patients. J Oral Rehabil. 2012;39(1):18-27.

24. Slade GD. Assessing change in quality of life using the Oral Health Impact Profile. Community Dent Oral Epidemiol. 1998;26(1):52-61. 
25. Aaronson NK, Muller M, Cohen PD, et al. Translation, validation, and norming of the Dutch language version of the SF-36 Health Survey in community and chronic disease populations. J Clin Epidemiol. 1998;51(11):1055-1068.

26. Ware JE Jr, Sherbourne CD. The MOS 36-item short-form health survey (SF-36). I. Conceptual framework and item selection. Med Care. 1992;30(6):473-483.

27. Ware JE Jr. SF-36 health survey update. Spine (Phila Pa 1976). 2000; 25(24):3130-3139.

28. Donner A, Klar N. Methods for comparing event rates in intervention studies when the unit of allocation is a cluster. Am J Epidemiol. 1994;140(3):279-289.

29. Campbell MK, Piaggio G, Elbourne DR, Altman DG; CONSORT Group. Consort 2010 statement: extension to cluster randomised trials. BMJ. 2012;345:e5661.

30. Maxwell SE, Delaney HD, Kelley K. Designing Experiments and Analyzing Data: A Model Comparison Perspective. $3^{\text {rd }}$ ed. Routledge; 2017.

31. Detels R, Tan CC. Oxford Textbook of Global Public Health. $6^{\text {th }}$ ed. Oxford University Press; 2015.

32. Durham J, Fraser HM, McCracken Gl, Stone KM, John MT, Preshaw PM. Impact of periodontitis on oral health-related quality of life. J Dent. 2013:41(4):370-376.
33. Gerdin EW, Einarson S, Jonsson M, Aronsson K, Johansson I. Impact of dry mouth conditions on oral health-related quality of life in older people. Gerodontology. 2005;22(4):219-226.

34. American Diabetes Association. Standards of Medical Care in Diabetes-2019 abridged for primary care providers. Clin Diabetes. 2019; 37(1):11-34.

35. Sedgwick P, Greenwood N. Understanding the Hawthorne effect. BMJ. 2015;351:h4672.

36. Macek MD, Tomar SL. Dental care visits among dentate adults with diabetes and periodontitis. J Public Health Dent. 2009;69(4): 284-289.

37. Tomar SL, Lester A. Dental and other health care visits among U.S. adults with diabetes. Diabetes Care. 2000;23(10):1505-1510.

38. Chaudhari M, Hubbard R, Reid RJ, et al. Evaluating components of dental care utilization among adults with diabetes and matched controls via hurdle models. BMC Oral Health. 2012;12:20.

39. Luo H, Bell RA, Wright W, Wu Q, Wu B. Trends in annual dental visits among US dentate adults with and without self-reported diabetes and prediabetes, 2004-2014. J Am Dent Assoc. 2018;149(6): 460-469.

\section{Get the Annals of \\ FAMILY MEDICINE} Family Medicine by E-mail

Make sure you see every new issue

while it's fresh; have the table of

contents sent to you by e-mail for

easy access to articles of interest.

Don't miss important research.

Request the e-mail table of contents at https://www.annfammed.org/

\section{JANUARYFFEBRUARY 2021 ․ Vol 19, No. 1}

Editorial | Original Research | Research Briefs I Special Reports । Reflections Innovations I Departments

Our January 2021 issue focuses on Racism and features a joint edlitorial from the editors of 10 North American primary care journals.

For more open access content, see our Racism in Medicine bibliography and preprint colloction.

The full text of the journal is available online at wmw. AnnFamMed.org, and through various aggregators, including PubMed Central, EBSCO, and Clinicalkey. The Annals is indexed in the MEDLINE, Science Citation Index Expanded, Current Contents/Clinical Medicine, EMBASE, and CINAHL databases.

\section{EDITORIAL}

Systemic Racism and Health Disparities: A Statement From Editors of Family Medicine Joumal's Sumi M. Sexton; Caroline R. Richardson; Sarina B. Schrager, Marjorie A. Bowman; John Hickner, Christopher P. Morley; Timothy F. Mott; Nicholas Pimlott; John W. Saultz; Barry D. Weiss

The Annals and 10 other leading North American primary care joumals pledge to contront the problem of racism in medicine by cultivating content that emphasizes cultural humility diversity 8 inclusion, and the racism in reflections on the theme of systemic racism and health inequitios.

\section{ORIGINAL RESEARCH}

ACC Discrimination and Medical Mistrust in a Racially and Ethnically Diverse Sample of Calffornia Adults Mohsen Bazargan; Sharon Cobb; Shervin Assar

The authors show that discrimination against patients, perceived or actual, is correlated with medical mistrust, and that decreasing discrimination may improve trust in medical providers and decrease dispartiles in heatth 